\title{
Mechanisms of visual threat detection in specific phobia
}

\author{
Mariann R. Weierich ${ }^{1,2}$ and Teresa A. Treat ${ }^{3}$ \\ ${ }^{1}$ Department of Psychology, Hunter College, The City University of New York, New York, NY, USA \\ ${ }^{2}$ The Graduate Center, The City University of New York, New York, NY, USA \\ ${ }^{3}$ Department of Psychology, University of Iowa, Iowa City, IA, USA
}

\begin{abstract}
People with anxiety or stress-related disorders attend differently to threat-relevant compared with non-threat stimuli, yet the temporal mechanisms of differential allocation of attention are not well understood. We investigated two independent mechanisms of temporal processing of visual threat by comparing spider-phobic and non-fearful participants using a rapid serial visual presentation task. Consistent with prior literature, spider phobics, but not non-fearful controls, displayed threat-specific facilitated detection of spider stimuli relative to negative stimuli and neutral stimuli. Further, signal detection analyses revealed that facilitated threat detection in spider-phobic participants was driven by greater sensitivity to threat stimulus features and a trend towards a lower threshold for detecting spider stimuli. However, phobic participants did not display reliably slowed temporal disengagement from threat-relevant stimuli. These findings advance our understanding of threat feature processing that might contribute to the onset and maintenance of symptoms in specific phobia and disorders that involve visual threat information more generally.
\end{abstract}

Keywords: Attention; Threat; Anxiety; Sensitivity; Attentional blink.

Most models of anxiety and stress-related conditions implicate preferential visual processing of threat-relevant information in the onset and maintenance of symptoms (e.g., Mathews \& MacLeod, 1994; Mogg \& Bradley, 2003). Over the past 15 years, there has been an increased emphasis on isolating the specific mechanisms through which threat-relevance affects the allocation of visual attention (e.g., Fox, Russo, Bowles, \& Dutton, 2001; Weierich, Treat, \& Hollingworth, 2008). Much of this considerable body of work has focused on spatial allocation of attention to threat (i.e., movement of attention towards or away from the location of threat information). However, spatial shifts are not the only mechanisms of interest for the operation of attention in anxiety and stress-related disorders. Once a location is attended, regardless of the efficiency of the initial spatial shift to that location, the features of threat-relevant information within the central visual field might be encoded more efficiently, or facilitated, thereby increasing the likelihood of triggering symptoms. In addition,

Correspondence should be addressed to: Mariann R. Weierich, Department of Psychology, Hunter College, The City University of New York, 695 Park Avenue, New York, NY 10065, USA. E-mail: mariann.weierich@hunter.cuny.edu

We wish to thank Brian J. Scholl for his input on this paper.

This work was supported by the National Institutes of Health under NIMHD [grant number MD007599]. 
when threat-relevant information is no longer present at a location, an anxious person might experience delayed temporal disengagement from the visual representation of that object, which could prolong anxious arousal. We examine two independent mechanisms of temporal allocation of attention to threat in anxiety: facilitated detection of visual threat features, and delayed temporal disengagement of attention from visual threat features. We accomplish this by leveraging a welldeveloped measurement approach for examining the subcomponent processes of visual attention in psychopathology, and by incorporating signal detection methods to further characterise these mechanisms.

In normative visual processing, identification of a first target (T1) within a stream of rapidly presented items in the same location impairs the detection of a subsequent target (T2) within approximately a 100-500 ms window (e.g., MacLean \& Arnell, 2012; Martin \& Shapiro, 2008). The period during which T2 detection is reduced is termed the "attentional blink" (AB). A number of theories recently have converged around the notion that processing of the first target activates a number of concurrent processes, and that innate capacity constraints impair second target detection (e.g., Chun \& Potter, 1995; Dux \& Marois, 2009; Raymond, Shapiro, \& Arnell, 1992). The basic visual effect (i.e., delayed detection of single letter T2 stimuli; Raymond et al., 1992) also can be influenced by the affective state of the perceiver. For example, dysphoric individuals displayed a greater basic $\mathrm{AB}$ effect than nondysphoric individuals (Rokke, Arnell, Koch, \& Andrews, 2002).

A growing body of research has focused on the degree to which the affective value of target stimuli, including anxiety- or threat-relevance, influences the $\mathrm{AB}$. When threat-relevant stimuli replace simple letter stimuli in a rapid serial visual presentation (RSVP) task, we can obtain an index of temporal allocation of attention to threatrelevant features. When $\mathrm{T} 1$ is threat-relevant, we might expect more difficulty with temporal disengagement from $\mathrm{T} 1$, evidenced by greater magnitude and/or duration of the blink compared to a non-threatening $\mathrm{T} 1$. In addition, we might expect facilitated detection of threat-relevant T2 stimuli, which would interrupt on-going processing of a neutral T1 and attenuate the magnitude and/or duration of the AB. Prior studies have examined either the facilitation of attention to or delayed disengagement from affective targets. No single study has investigated both processes in the same participants, and task parameters vary for each study, so we do not yet have a comprehensive understanding of the temporal mechanisms underlying processing of threat features.

There is some evidence of facilitated detection of affective T2 stimuli in anxiety, as indexed by an attenuated AB. For example, spider phobics correctly detected spider photos in a predictable T2 position more often than positive, neutral, negative, and snake photos, whereas non-fearful controls correctly detected spider T2s less often than the other stimulus categories (Trippe, Hewig, Heydel, Hecht, \& Miltner, 2007). In another study, spider-fearful participants did not show significantly greater detection of T2 spiders (Reinecke, Rinck, \& Becker, 2008); however, overall T1 performance was exceptionally low, suggesting that an unusually challenging T1 task might have altered $\mathrm{T} 2$ processing to an unknown degree.

Other RSVP studies of facilitated threat detection in anxiety have shown facilitated detection of fearful faces compared with happy faces in highstate anxiety (Fox, Russo, \& Georgiou, 2005), but also facilitated detection of angry faces in both high and low social anxiety (de Jong \& Martens, 2007). Participants unselected for anxiety also showed facilitated detection of angry schematic faces (Maratos, Mogg, \& Bradley, 2008). In addition to basic visual stimuli, people also show facilitated detection of affective semantic T2 stimuli including emotional words (Anderson, 2005; Anderson \& Phelps, 2001), negative Japanese kanji characters (Ogawa \& Suzuki, 2004), and arousing negative and positive verbs (Keil \& Ihssen, 2004). Overall, the literature suggests facilitated detection of affective information, and potentiation of this effect among trait- or state-anxious individuals.

Facilitated detection of affective T2 stimuli in anxiety often is framed as an effect of threat- 
relevance. However, the specific mechanism through which "threat" (compared with negative or generally affective information) drives enhanced perception has not been clearly delineated. A general response bias (i.e., a lower threshold to report the presence of an affective target) might account for the facilitated detection of affective words at T2 (Tibboel, Van Bockstaele, \& De Houwer, 2011). However, the effect in that study could have been due to more efficient processing of semantic stimuli, and might not generalise to threat images. In addition to a possible bias for threat image reporting at $\mathrm{T} 2$, anxious individuals also might have enhanced perceptual sensitivity to the visual features of threat stimuli. Such sensitivity could account for facilitated detection of threat-relevant T2 stimuli, and could correspond to heightened detection and reaction to threat or threat-like physical information in the real world.

The second mechanism of interest is temporal disengagement, or the degree to which threatrelevant stimuli interfere with the processing of subsequent non-threat stimuli in the same spatial location. Some studies show an attenuated $A B$ following threat-relevant semantic $\mathrm{T} 1$ stimuli in state anxiety (Arend \& Botella, 2002), a shorter blink following T1 spider words in spider fear (Cisler, Ries, \& Widner, 2007), and a reduced blink following trauma-relevant words in posttraumatic stress disorder (PTSD) (Amir, Taylor, Bomyea, \& Badour, 2009). One interpretation of these results is that anxious people process threatrelevant words more efficiently (e.g., Arend \& Botella, 2002). However, in other studies stateanxious participants also showed slowed disengagement from threat words (Barnard, Ramponi, Battye, \& Mackintosh, 2005), and participants unselected for anxiety showed slowed disengagement from highly arousing words (i.e., sex-related or taboo) at T1 (e.g., Arnell, Killman, \& Fijavz, 2007; Mathewson, Arnell, \& Mansfield, 2008). The mixed evidence for temporal disengagement from anxietyrelevant or negatively valenced stimuli might be a function of the use of word stimuli. Semantic processing requirements within a perceptual task introduce the possibility that some anxious individuals process threat words more or less efficiently, or that some individuals generally process negative or arousing words differently. Such findings are interesting but do not address the current question regarding how anxious individuals process threatrelevant percepts (e.g., actual spiders) in the world.

Some studies have investigated difficulty with disengagement from generally affective and threatrelevant images in the RSVP task. For example, evidence shows a greater blink following negatively arousing (Most, Chun, Widders, \& Zald, 2005), aversively conditioned (Smith, Most, Newsome, \& Zald, 2006), sexually arousing (Most, Smith, Cooter, Levy, \& Zald, 2007) and angry face (De Jong, Koster, Van Wees, \& Martens, 2009) $\mathrm{T} 1$ stimuli. In anxiety, a longer $\mathrm{AB}$ (i.e., slower temporal disengagement) has been shown in obsessive-compulsive disorder (OCD) following erotic images (Olatunji, Ciesielski, \& Zald, 2011), in PTSD following angry faces (Schonenberg \& Abdelrahman, 2013), and in combat PTSD following combat-threat images (Olatunji, Armstrong, McHugo, \& Zald, 2013). These results suggest that, consistent with the original task, perceptual stimuli produce stronger $\mathrm{AB}$ effects, and also that the arousal level of the stimuli may be more important than the valence. This notion is of particular importance in anxiety and stress disorders, as threat-relevant stimuli might not be objectively valenced (e.g., a cardboard box in the road), yet have acquired the properties of high arousal as well as negative valence for some individuals (e.g., a combat veteran with PTSD following a roadside bomb attack).

The aim of this study is to reconcile some of the inconsistencies in the literature examining the temporal facilitation of attention to and disengagement of attention from threat. We use spider phobia as a model condition, given the circumscribed and homogeneous nature of threat-relevant stimuli, and we investigated both facilitation and disengagement mechanisms within a single study, to compare directly the threat-linked temporal characteristics of both processes.

We tested two primary hypotheses. First, we expected phobic individuals to display enhanced temporal facilitation of attention to threat-relevant T2 stimuli (i.e., greater threat T2 detection) relative to controls. Second, we expected phobic

\section{COGNITION AND EMOTION, 2015, 29 (6)}


individuals to show greater difficulty disengaging attention temporally from threat-relevant $\mathrm{T} 1$ stimuli, in comparison to controls. Both the hypothesised facilitation and disengagement effects would be independent of baseline processing and any effect of negative valence.

In addition, to further investigate the subcomponent processes driving differential detection of threat features, we used signal detection theory (SDT) methods to evaluate the degree to which the hypothesised facilitated threat detection effects could be attributable to perceptual sensitivity or decision bias. Greater facilitated detection of threat could reflect either (1) an enhanced ability to discriminate threat and non-threat features; (2) a lowered decision threshold for detection of threatening stimuli or (3) both greater sensitivity and a more liberal threshold. One study did not provide evidence of bias (i.e., lowered threshold) for detection of affective (taboo) word stimuli in unselected participants (Tibboel et al., 2011). In another study, spider phobics showed a lower spider detection threshold but not greater sensitivity, relative to controls (Becker \& Rinck, 2004). However, in the latter study, task instructions might have artificially enhanced controls' attention to spiders, as spider stimuli were presented in a separate block with the instruction to indicate the presence or absence of a spider only. The present study tested the degree to which facilitated detection of threat-relevant information in specific phobia is attributable to both perceptual and decisional aspects of processing and, if so, whether these effects are threat-specific and independent of negative valence.

\section{METHOD}

\section{Participants}

Twenty-five spider-phobic and 31 non-fearful control participants aged 18-30 years (64\% female, $36 \%$ male; self-reported ethnicity 66\% White, $14 \%$ Black, 7\% Hispanic or Latino/a, 9\% Asian, 2\% American Indian, 2\% other ethnicity) were recruited via an introductory psychology subject pool, campus fliers and email recruitment to the students of a north-eastern US university. Subject pool participants were selected based on their responses to a pre-screening questionnaire as part of a larger pre-screening packet. Participants who responded to the flier and the email recruitment completed the pre-screening questionnaire online. All human subjects study procedures were approved by the institutional review board. We report how we determined our sample size, all data exclusions, all manipulations and all measures in the study.

\section{Pre-screening}

The pre-screening measure included a subset of 10 items from the 18-item Fear of Spiders Questionnaire (FSQ; Szymanski \& O'Donohue, 1995) and a subset of 10 items from the 30-item Snake Anxiety Questionnaire (SNAQ; Klorman, Weerts, Hastings, Melamed, \& Lang, 1974). Based on the literature utilising the full scales (e.g., Muris \& Merckelbach, 1996), we set the pre-screening cutoff for spider fear at a minimum FSQ subset score of 45 (range: $0-60$ ). This conservative limit on the abbreviated measure corresponds to the full-scale clinical sample mean minus .5 $S D(M=89, S D=$ 19; Muris \& Merckelbach, 1996). To avoid confounding negative valence with animal fear, we set the maximum pre-screen SNAQ subset score at 2 (range: 0-10). Non-fearful control participants' pre-screen scores for spider and snake fear both were $\leq 2$. We based our a priori power analysis on the large effect sizes reported in the blink literature (e.g., Most et al., 2005). For a large effect (i.e., $d=.8$ ), $\alpha=.05$, and expected sample means and standard deviations (the literature are consistent regarding the range of expected T2 per cent correct detection), 18 participants in each group provide adequate power.

\section{Questionnaire/interview measures}

Following the experimental tasks, participants completed self-report measures that included the full FSQ, the full SNAQ, Fear Questionnaire (FQ) items relevant to snake and spider fear (Marks \& Mathews, 1979), the State-Trait Anxiety Inventory State version (Spielberger, Gorsuch, 
\& Lushene, 1970) and the Beck Depression Inventory II (Beck, Steer, \& Brown, 1996). The experimenter also conducted Structured Clinical Interview for the Diagnostic and Statistical Manual of Mental Disorders, 4th Edition (DSM-IV) (SCID-IV) modules for major depression, psychotic symptoms and phobic symptoms.

Data from participants were excluded if fullscale scores on spider measures did not meet threshold for spider phobia. We developed the spider fear criterion based on the scores reported in a clinical sample of spider-phobics $(M=89, S D$ = 19; Muris \& Merckelbach, 1996); we excluded participants whose FSQ scores were below 1.5 SD of the clinical mean. We also selected FQ cut-offs based on their correspondence with diagnostic criteria (i.e., avoidance and distress/impairment) for specific phobia. Thus, the criteria were set at FSQ score $\leq 61$; FQ avoidance of spider item $\leq 6$ (i.e., "markedly avoid"); FQ present state of phobia item $\geq 3$ (i.e., "definitely disturbing or disabling”). Spider-phobic participants also were required to endorse criteria for phobia during the administration of the SCID-specific phobia module. Finally, we included only spider phobics and control participants who scored $\leq 15$ on the $\mathrm{SNAQ}_{2}$ in order to ensure that reported fear of snakes was not in the snake phobic range. Data from four participants were excluded due to below threshold spider fear scores on the FSQ or nonendorsement of phobia on the SCID.

\section{Materials}

We chose task parameters to address some of the limitations of prior studies. First, we utilise photo stimuli to enhance generality to the experience of people with spider phobia in the world and to measure perceptual processing without the extra element of semantic processing. Second, prior studies typically compare threat with neutral stimuli, thus introducing a potential valence confound. We therefore compare stimuli from three non-overlapping categories: threat, non-threat negative, and neutral, thus allowing disambiguation of the effects of threat, valence and baseline perceptual processing.

\section{COGNITION AND EMOTION, 2015, 29 (6)}

We used 26 photo stimuli from each of six categories as target stimuli. Spider photos were the threat-relevant animal stimuli, snake photos were the negative, non-threat animal stimuli, frog photos were the neutral animal stimuli, and photos of apples, grapes and oranges were neutral, non-animal stimuli. We sized all target objects to fill the image space as much as possible. Distractor stimuli were photos of plants and trees. All photos were drawn from non-copyrighted photographs on the Internet, and the animal photos specifically were selected such that each group contained photos with similar colour composition and luminance.

\section{Task design}

The RSVP task was programmed using E-Prime software and presented on a Dell Dimension 8100 desktop with an Intel Pentium 4 processor using a Dell P991 CRT monitor set to a refresh rate of $100 \mathrm{~Hz}$. Stimulus photos were $6 \mathrm{~cm}$ high and 6 $\mathrm{cm}$ wide, and they were presented at fixation on a black screen. Target and distractor stimuli were randomly selected for each trial. Each RSVP photo stream (Figure 1) contained 18-20 photos.

The first target (T1) in each stream was preceded randomly by 8 or 10 distractor photos. Half of the trials also contained second targets (T2), which appeared randomly in one of three positions, or lags, after the T1 image. Because our goal was to test stimulus-related differences in $\mathrm{T} 2$ detection, rather than mapping the full $\mathrm{AB}$ effect, we enhanced our power by focusing on a subset of lags. Based on pilot data, we selected lags 2 and 3 as the lags most likely to reflect decreased T2 performance (i.e., early lags following T1). The third lag for each block was a later lag (i.e., 550$600 \mathrm{~ms}$ following T1), chosen as a lag less likely to be affected by the $\mathrm{AB}$ phenomenon. Each photo stimulus type appeared 12 times at each lag for each of the two conditions (i.e., the disengagement block and the facilitation block). One additional randomly selected distractor photo was presented at the end of each stream to mask T2 images that were presented at the last lag. 
a

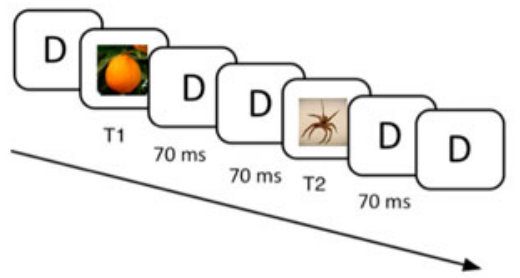

Facilitation trial b

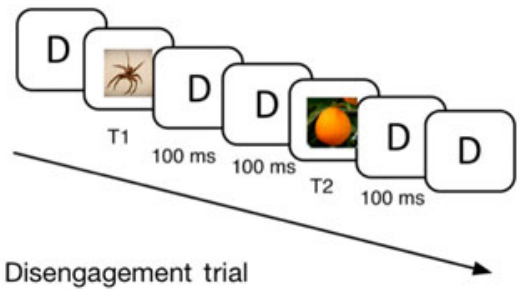

Figure 1. (a) Section of a single facilitation trial of the RSVP paradigm; the threat stimulus in the second target position (T2) appears at lag 3, or $210 \mathrm{~ms}$ after the fruit stimulus in the first target position (T1). (b) Section of a single disengagement trial of the RSVP paradigm, with the threat stimulus in the first target position (T1) and the fruit stimulus in the second target position (T2). $D=$ non-target distractor. Photographic images retrieved via fickr.com under Creative Commons license https://creativecommons.org/ licenses/by/2.0/legalcode. Fruit image (Pamela Carls) and spider image (Dan Valentine) resized with no other modification.

\section{Procedure}

After consent, participants sat in a comfortable chair approximately $70 \mathrm{~cm}$ from the monitor in a dimly lit, sound attenuated room. Participants completed a total of 452 trials (10 practice plus 216 trials per block) of the RSVP paradigm. Block type was counterbalanced across participants.

\section{Facilitation trial block}

For facilitation trials, we measured the efficiency of affective (threat, negative, and neutral) T2 target detection. Each RSVP stream in this block contained neutral (fruit) target stimuli in the T1 position, and an animal appeared in the $\mathrm{T} 2$ position for T2-present trials. Each photo within the stream was presented for $70 \mathrm{~ms}$; this was the timing parameter that most consistently produced the $\mathrm{AB}$ in pilot work. ${ }^{1} \mathrm{We}$ used lags 2,3 and 8 in this block, which corresponded to 140, 210 and $560 \mathrm{~ms}$ after T1, respectively.

A text screen clearly stating the tasks for each block and the order of target stimuli preceded each block. For the facilitation block, after each stream participants were asked to identify the fruit (i.e., "apple," "grape" or "orange") by pressing the corresponding key, and to indicate whether an animal was present or absent, also by pressing a key. Participants then pressed the spacebar to begin the next trial. Responses were not timed.

\section{Disengagement trial block}

For disengagement trials, we measured the magnitude of the AB following affective T1 stimuli. This block was identical to the facilitation block, with the exception of target order and SOA. Based on the pilot data, each photo within the RSVP streams was presented for $100 \mathrm{~ms}$ in this block. We presented T2 stimuli at lags 2, 3 and 6, or 200, 300 and $600 \mathrm{~ms}$ after T1. For the disengagement block, animal stimuli appeared in the T1 position, and fruit appeared in the $\mathrm{T} 2$ position on $\mathrm{T} 2$ present trials. After each stream, participants were asked to identify the animal (i.e., "spider," "snake" or "frog") by pressing the corresponding key, and to indicate whether fruit was present or absent, also by pressing a key. Participants then pressed the spacebar to begin the next trial.

\footnotetext{
${ }^{1}$ The stimulus onset asynchronies (SOAs) used in the original AB studies (i.e., 90 ms SOA; Raymond, et al., 1992; Shapiro et al., 1994) were calibrated for the speed necessary to process single letter stimuli. Given the perceptual differences between our photo stimuli and the original letter stimuli, we piloted the facilitation and disengagement tasks to determine the optimal SOA for each. Initial piloting of the facilitation task at $100 \mathrm{~ms}$ SOA revealed ceiling effects in non-fearful pilot participants. We therefore decreased the SOA by $10 \mathrm{~ms}$ increments until pilot participants performed at an average of $65 \%$ correct T2 detection at the lags most likely to show the effect (i.e., early lags). For the disengagement task, $100 \mathrm{~ms} \mathrm{SOA}$ was optimal; we attribute this difference to the perceptual similarity between the fruit $\mathrm{T} 2$ targets and the distractors in the disengagement task (i.e., the greater difficulty of the disengagement task).
} 


\section{Questionnaire/interview}

Following the experimental task, participants completed the questionnaires and the brief interview. The experimenter then debriefed each participant and provided payment (\$15) or credit (1.5 introductory psychology experiment credits).

\section{RESULTS}

\section{Participant characteristics}

There were more female participants in the phobia group (81\%) than in the control group (52\%), consistent with the higher prevalence of spider phobia among women (e.g., Fredrikson, Annas, Fischer, \& Wik, 1996). The groups did not differ on age or ethnicity. For spider fear and state anxiety, spider-phobic participants' scores were significantly higher than control participants (Table 1).

No participants reported psychotic symptoms during the SCID interview. Six participants (three phobics and three controls) endorsed moderate levels of depression during the interview; these participants' experimental data did not differ from those of the non-depressed participants, so we retained the data.

\section{T1 performance on RSVP task}

The primary dependent variable of interest (i.e., T2 detection accuracy) is predicated on correct $\mathrm{T} 1$ identification, as the $\mathrm{AB}$ phenomenon requires processing of a first target that subsequently interferes with processing of a second target (e.g., Raymond et al., 1992). Participants correctly identified T1 stimuli in $80 \%(S D=8 \%)$ of facilitation trials and 93\% $(S D=5 \%)$ of disengagement trials. For disengagement trials, T1 identification accuracy was lower for frogs $\left(M_{\mathrm{ACC}}=91 \%, S D=7 \%\right)$ compared with snakes $\left(M_{\mathrm{ACC}}=94 \%, S D=9 \%\right.$; $t(55)=2.98, p<.05, d=.36)$ and spiders $\left(M_{\mathrm{ACC}}=\right.$ $96 \%, S D=4 \% ; t(55)=6.55, p<.001, d=.92) . \mathrm{T} 1$ identification accuracy also was lower for snakes compared with spiders $(t(55)=5.06, p<.001, d=$ .68). Despite these statistically significant differences in the full sample, T1 identification was greater than $90 \%$ across all animal types, and there were no significant group differences in $\mathrm{T} 1$ identification for any $\mathrm{T} 1$ category, all $p$ s $>.05$. Only trials with accurate $\mathrm{T} 1$ identification were included in analyses. Per cent correct T2 detection data given accurate $\mathrm{T} 1$ are presented in Table 2 .

\section{Facilitation of threat detection}

We conducted a repeated measures analysis of variance (ANOVA) with Lag (140, 210 and 560 $\mathrm{ms}$ ) and Animal (spider, snake and frog) as within subjects factors, and Group (phobic and nonfearful) as the between subjects factor. The first step in the analysis was to establish the presence of the $\mathrm{AB}$ effect. This was confirmed by a main effect of lag $(F(2,108)=7.49, M S E=.029, p=.001$, $\left.\eta_{\mathrm{p}}^{2}=.122\right)$. Follow-up tests showed that overall per cent correct T2 detection at both early lags (lag $2 M$ $=.67, S D=.14$; lag $3 M=.65, S D=.18$; ) was

Table 1. Participant characteristics

\begin{tabular}{|c|c|c|c|c|}
\hline Measure & $\begin{array}{c}\text { Spider-fearful }(\mathrm{n}=25) \\
\mathrm{M}(S D)\end{array}$ & $\begin{array}{c}\text { Non-fearful controls }(\mathrm{n}=31) \\
\mathrm{M}(S D)\end{array}$ & $\mathrm{t}(54)$ & Cohen's d \\
\hline FSQ & $80.9(15.5)$ & $7.8(11.4)$ & $20.32^{*}$ & 5.37 \\
\hline FQ1 (avoid spider) & $6.4(1.0)$ & $1.1(1.0)$ & $19.46^{*}$ & 5.30 \\
\hline FQ2 (avoid snake) & $1.6(1.3)$ & $1.9(1.2)$ & 1.02 & -.24 \\
\hline FQ3 (rate fear) & $4.6(1.8)$ & $1.1(1.0)$ & $9.37^{*}$ & 2.40 \\
\hline SNAQ & $5.0(4.8)$ & $4.1(3.2)$ & 0.74 & .22 \\
\hline STAI-S & $40.0(9.1)$ & $30.4(10.2)$ & $3.69^{* *}$ & .99 \\
\hline BDI-II & $11.1(8.4)$ & $7.2(7.4)$ & 1.83 & .49 \\
\hline
\end{tabular}

Note: FSQ, Fear of Spiders Questionnaire, range: 0-108; FQ, Fear Questionnaire, range: 0-8; SNAQ, Snake Anxiety Questionnaire, range: 0-30; STAI-S, State-Trait Anxiety Inventory, State Version; BDI-II, Beck Depression Inventory II. ${ }^{*} p<.001 ;{ }^{* *} p<.01$. 
Table 2. Per cent correct (SD) T2 detection given correct T1 identification

\begin{tabular}{|c|c|c|c|c|c|c|c|c|c|}
\hline \multicolumn{10}{|c|}{ Facilitated detection } \\
\hline T2 Type & \multicolumn{3}{|c|}{ Threat (spider) } & \multicolumn{3}{|c|}{ Negative (snake) } & \multicolumn{3}{|c|}{ Neutral (frog) } \\
\hline$L a g^{\mathrm{a}}$ & $\operatorname{Lag} 2$ & $\operatorname{Lag} 3$ & $\operatorname{Lag} 8$ & $\operatorname{Lag} 2$ & $\operatorname{Lag} 3$ & $\operatorname{Lag} 8$ & $\operatorname{Lag} 2$ & $\operatorname{Lag} 3$ & $\operatorname{Lag} 8$ \\
\hline Phobic & $84(1)$ & $84(1)$ & $83(1)$ & $78(2)$ & $75(2)$ & $85(1)$ & $59(2)$ & $61(2)$ & $59(2)$ \\
\hline Control & $66(2)$ & $58(3)$ & $71(2)$ & $69(2)$ & $66(2)$ & $76(2)$ & $52(2)$ & $49(2)$ & $63(2)$ \\
\hline \multicolumn{10}{|c|}{ Disengagement } \\
\hline T1 Type & \multicolumn{3}{|c|}{ Threat (spider) } & \multicolumn{3}{|c|}{ Negative (snake) } & \multicolumn{3}{|c|}{ Neutral (frog) } \\
\hline $\operatorname{Lag}^{\mathrm{b}}$ & $\operatorname{Lag} 2$ & $\operatorname{Lag} 3$ & $\operatorname{Lag} 6$ & $\operatorname{Lag} 2$ & $\operatorname{Lag} 3$ & $\operatorname{Lag} 6$ & Lag 2 & $\operatorname{Lag} 3$ & $\operatorname{Lag} 6$ \\
\hline Phobic & $65(2)$ & $75(2)$ & $89(1)$ & $69(2)$ & 81(1) & $90(1)$ & $74(2)$ & $78(2)$ & $91(1)$ \\
\hline Control & $66(2)$ & $74(2)$ & $89(1)$ & $66(3)$ & $78(2)$ & $90(1)$ & $65(2)$ & $74(2)$ & 89(1) \\
\hline
\end{tabular}

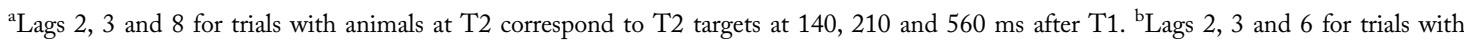
animals at T1 correspond to T2 targets at 200, 300 and $600 \mathrm{~ms}$ after T1.

significantly lower than T2 detection at the late lag (lag $8 M=.72 ; S D=.13 ;$ lag 2 vs. lag $8, t(55)=$ $-2.82, p<.01$; lag 3 vs. lag $8, t(55)=-3.58, p<$ .01). The $\mathrm{AB}$ effect was evident at both early lags, and T2 detection at lag 2 and lag 3 did not differ from each other. We therefore averaged lags 2 and 3 to investigate the magnitude and characteristics of the $\mathrm{AB}$ in all subsequent facilitation analyses. All reported $p$ values are two-tailed.

\section{Per cent correct analyses}

We calculated percentage of correct $\mathrm{T} 2$ detection for T2-present trials (i.e., per cent correct hits). There was a main effect of Group in the omnibus repeated measures ANOVA: overall per cent correct T2 detection for spider phobics $(M=.74$; $95 \%$ CI $[.69, .79])$ was higher than that of controls $(M=.63,95 \%$ CI $[.59, .68]),(F(1,54)$ $\left.=11.31, M S E=.128, p=.001 \eta_{\mathrm{p}}^{2}=.173\right)$. There was also a main effect of Animal $(F(2,108)=$ $\left.52.40, M S E=.032, p<.001, \eta_{\mathrm{p}}^{2}=.49\right)$, driven by better overall T2 detection for snakes $(M=.75$, $95 \% \mathrm{CI}[.71, .79])$ and spiders $(M=.74,95 \% \mathrm{CI}$ $[.70, .78])$ compared with frogs $(M=.57,95 \% \mathrm{CI}$ $[.53, .61])$. A significant Group by Animal $(F(2$, $\left.108)=6.421, M S E=.032, p<.01, \eta_{\mathrm{p}}^{2}=.106\right)$ interaction was driven by better T2 detection of spiders $(M=.84,95 \% \mathrm{CI}[.77, .89])$ in the phobic group compared with the control group $(M=.65$, $95 \%$ CI $[.59, .70])$, and a significant Group by Lag $(F(2,108)=3.151, M S E=.029, p<.05$, $\left.\eta_{\mathrm{p}}^{2}=.055\right)$ interaction was driven by better $\mathrm{T} 2$ detection at lags 2 and 3 in the phobic group (lag $2 M=.74,95 \%$ CI $[.68, .77]$; lag $3 M=.73,95 \%$ CI $[.66, .80])$ compared with the control group (lag $2 M=.62,95 \%$ CI $[.57, .67]$; lag $3 M=.58$, 95\% CI $[.52, .64])$.

Given non-task-related individual differences in basic perceptual processing during the $\mathrm{AB}$ (e.g., Klein, Arend, Beauducel, \& Shapiro, 2011), which were demonstrated in the main effect of Group and the Group by Lag interaction, it is necessary to control for each subject's baseline T2 detection performance. The second step in our analytic strategy thus was to conduct planned comparisons to investigate whether phobics demonstrated an advantage in detecting phobia-relevant stimuli, controlling for their baseline perceptual processing of neutral and negative stimuli. We computed the difference in T2 accuracy for spider and frog stimuli ("threat-neutral"), and the difference in T2 accuracy for spider and snake stimuli ("threat-negative"; see Figure 2). We then conducted planned comparisons based on our hypotheses to evaluate whether spider phobics showed significantly greater difference scores (i.e., greater detection of threat beyond neutral and negative stimuli detection) 


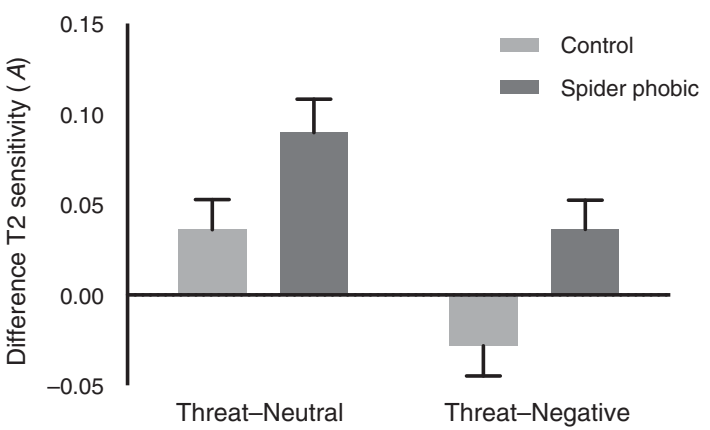

Figure 2. For facilitation trials, difference scores representing average sensitivity (lags 2 and 3) to threat (spider) stimuli controlling for sensitivity to neutral (frog) stimuli in the left columns and negative (snake) stimuli in the right columns. Greater values represent enhanced sensitivity to features of spiders at compared with other types of stimuli at T2. Error bars represent standard errors.

than controls. Compared with controls, phobic participants displayed greater per cent correct T2 detection of phobia-relevant stimuli controlling for detection of neutral T2 stimuli, $t(54)=2.62, p=$ .011 , Hedge's $g$ (bias-corrected effect size) $=.67$, and for phobia-relevant stimuli controlling for detection of negative T2 stimuli, $t(54)=2.60, p=$ .012 , Hedge's $g=.69$. Spider phobics, in comparison to controls, demonstrated greater facilitated detection of threat-relevant stimuli than negative or neutral stimuli.

\section{Sensitivity analyses}

The third step in our analysis was to decompose the basic blink effect into its subcomponents based on signal detection theory (SDT). We decomposed the variability in $\mathrm{T} 2$ accuracy into sensitivity (i.e., the ability to detect the presence of T2 features) and bias (i.e., the threshold at which participants determined that a T2 was present). We computed $A$, a non-parametric sensitivity measure that estimates sensitivity more accurately than other nonparametric measures (Zhang \& Mueller, 2005). A ranges from 0 to 1.0 , where .5 indicates null sensitivity [i.e., equal number hits and false alarms $(\mathrm{FAs})]$ and 1.0 indicates perfect sensitivity (i.e., $100 \%$ hits, 0\% FAs). The FA rate in our analyses was each participant's global FA rate, or tendency to report the presence of any $\mathrm{T} 2$ target on targetabsent trials.
To evaluate sensitivity to phobic stimuli controlling for general perceptual sensitivity, we computed the differences between sensitivity $(A)$ for spider stimuli and sensitivity for snake and frog stimuli (Figure 2). Planned comparisons paralleled the per cent correct T2 detection results. Phobics' sensitivity to threat-relevant stimuli controlling for sensitivity to neutral stimuli was greater than that of control participants, $t(54)=2.67, p=.010$, Hedge's $g=.70$. Similarly, phobics' sensitivity to threatrelevant stimuli controlling for negative stimuli was greater than that of controls, $t(54)=2.13, p=.038$, Hedge's $g=.66$, indicating that spider phobics', but not controls', sensitivity to threat stimuli was independent of negative valence or baseline (neutral) sensitivity. Thus, one mechanism by which spider phobics display facilitated threat detection is enhanced sensitivity to the perceptual features of threat objects.

\section{Threshold analyses}

We were also interested in the degree to which bias accounted for the differences in T2 accuracy between groups; if spider-phobic individuals require less perceptual information to make the decision that they have seen a spider in the second target position, better performance for $\mathrm{T} 2$ spider detection in phobics might be due to a lowered or more liberal perceptual threshold for those participants. We computed the non-parametric bias measure $B^{\prime \prime}$ for each participant's T2 performance for each type of stimulus. Positive values of $B^{\prime \prime}$ denote a bias towards responding "T2 absent" whether T2 is present or not (i.e., a more conservative threshold), whereas negative values of $B^{\prime \prime}$ denote a bias towards responding "T2 present" (i.e., a more liberal threshold), whether T2 is present or not (Figure 3). As with the sensitivity analyses, we used each participant's global FA rate (i.e., tendency to respond $\mathrm{T} 2$ present in the absence of a target) and correct hits by stimulus type for the bias calculation.

We computed differences between the threshold for spiders and thresholds for snakes and frogs, to examine group differences in spider-detection thresholds controlling for snake and frog detection thresholds. Phobics, for example, showed an 


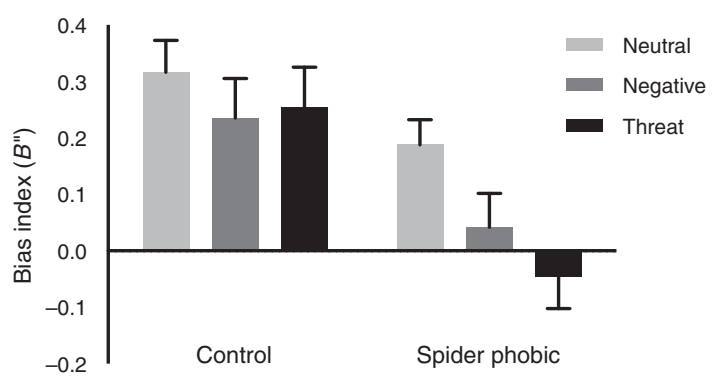

Figure 3. For facilitation trials, average bias (lags 2 and 3) by stimulus type by group. Negative values represent a more liberal bias for reporting the presence of a T2 stimulus, and positive values represent a more conservative bias for reporting the absence of a $\mathrm{T} 2$ stimulus. Error bars represent standard errors.

average difference score for the "threat-neutral" comparison of -.23 , indicating that their threshold for spiders was .23 points lower (i.e., more liberal) than their threshold for frogs. In contrast, controls showed an average "threat-neutral" average score of -.06 , indicating that their spider threshold was only .06 points more liberal than their frog threshold. Consistent with these descriptive statistics, a planned comparison revealed that spider phobics displayed a significantly more liberal threshold for spiders than neutral stimuli, relative to controls, $t(54)=2.98, p=.004$, Hedge's $g=.80$. Spider phobics also showed a more liberal threshold for spider stimuli than for negative stimuli, in comparison to controls, $t(54)=2.06$, $p=.044$, Hedge's $g=.53$. Thus, phobics' greater detection of threat-relevant information also can be attributed in part to a more liberal threshold for the detection of threatening stimuli.

\section{Disengagement of attention from threat}

The fourth step in our analysis was to investigate the degree to which threat-relevant $\mathrm{T} 1$ stimuli might impact the magnitude of the $\mathrm{AB}$ as measured by detection of neutral T2 stimuli. We conducted an omnibus repeated measures ANOVA with Lag (200, 300 and $600 \mathrm{~ms}$ ) and T1 Animal (spider, snake and frog) as within subjects factors and Group as the between subjects factor. Consistent with the presence of the basic blink effect, there was a main effect of Lag $(F$ $(1.71,92.53)=74.51, M S E=.032, p<.000$, $\eta_{\mathrm{p}}^{2}=.58$, with a Greenhouse-Geisser correction for the violation of sphericity). Overall per cent correct T2 accuracy rates at both early lags (lag 2 $M=.67, S D=.19 ;$ lag $3 M=.77, S D=.15$ ) were significantly lower than the T2 detection rate at the late lag (lag $6 M=.89, S D=.08$; lag 2 vs. lag $6, t(55)=-10.44, p<.001$; lag 3 vs. lag $6, t(55)=$ $-7.79, p<.001$ ), indicating the presence of the basic blink effect. Given the presence of the $A B$ at both early lags and to enhance power, we averaged across these lags for subsequent analyses. We note that although there were no other significant main effects or interactions in the omnibus test, our analytic plan is based on our a priori planned comparisons. Only analyses of per cent correct indices are presented below, as we did not make predictions regarding sensitivity to and biases for identification of neutral (i.e., fruit) $\mathrm{T} 2$ stimuli.

\section{Per cent correct}

To examine whether phobics showed slower disengagement from threat-relevant stimuli than from neutral and negative stimuli, we computed two difference scores. One quantified the difference in performance for threat and neutral stimuli ("threat -neutral"), and the other quantified the difference in per cent correct for threat and negative stimuli ("threat-negative"). Phobics and controls did not show reliable differences ${ }^{2}$ in their "threat-neutral"

\footnotetext{
${ }^{2}$ Per reviewer suggestion, we conducted exploratory ANOVAs separately at each of the early lags: there were no significant Animal x Group interactions. Also, given the possibility of habituation within a task block, we conducted exploratory ANOVAs for the first half of the trials within the disengagement block. At Lag 2 only there was a significant Animal $\times$ Group interaction $(p=.045)$. T-tests of differences at Lag 2 showed only a greater difference for Neutral-Threat (frog-spider) in phobics compared with controls $(p=.017)$. This is an interesting preliminary suggestion that phobics might disengage more slowly from threat relative to neutral on the first few encounters with threat stimuli, but there is no suggestion of threat specificity (Negative-Threat). However, this analysis was purely exploratory and runs the risk of Type I error.
} 
scores $(M \mathrm{~s}=.06$ and .00 , respectively), $t(54)=1.65$, $p=.104$, Hedge's $g=.44$, or in their "threat -negative" scores $(M \mathrm{~s}=.05$ and .02 , respectively), $t(54)=.826, p=.412$, Hedge's $g=.22$.

\section{DISCUSSION}

Consistent with hypotheses, spider-phobic participants demonstrated markedly enhanced detection of threat-relevant stimuli at early lags compared with non-fearful controls. The effect was specific to phobia-relevant stimuli and was not accounted for by baseline performance or by negative valence. Signal detection analyses of the facilitation data showed that phobic participants displayed enhanced sensitivity to spider stimuli compared with neutral and (at a trend level) negative stimuli. This result is inconsistent with Becker and Rinck's (2004) earlier demonstration that spider-phobic individuals do not display enhanced perceptual sensitivity to spider stimuli. However, Becker and Rinck directed participants' attention explicitly and specifically to spiders in a block of trials in which a spider either was or was not present (i.e., no other animal potentially was present). The absence of stimulus competition might have induced an attentional set for spider stimuli (Mathews \& Mackintosh, 1998), which could have also enhanced controls' sensitivity. Indeed, the visual system processes low level features relatively easily (e.g., Chaumon, Kveraga, Barrett, \& Bar, 2013; Chen, 2012) and the efficiency of such low level processing is directly related to associative learning (Panichello, Cheung, \& Bar, 2013), such that an attentional set could enhance performance.

Signal detection analyses also revealed that phobic participants displayed a lower detection threshold (liberal response bias) for spider stimuli. This result is consistent with Becker and Rinck (2004), who found that spider-phobic individuals displayed a more liberal response bias than controls for both spider and beetle stimuli. Our results further demonstrate that phobics' lowered perceptual threshold for spiders was not attributable to differential processing of negatively valence (i.e., snakes), and that the lowered perceptual threshold was robust to stimulus competition.

Counter to hypotheses, phobic participants did not display reliably greater difficulty than control participants with temporal disengagement from threat-relevant stimuli compared with neutral or negative stimuli. This finding is inconsistent with the growing literature demonstrating normatively slowed disengagement from negatively or positively arousing T1 images (e.g., Most et al., 2005, 2007; Smith et al., 2006). Given that spider phobics find spider photos to be more arousing (and negative) than controls, we had anticipated that phobics would display decreased $\mathrm{T} 2$ detection following spider T1 stimuli.

There are several potential explanations for the absence of a statistically reliable disengagement effect. Although one of the primary strengths of the current study is our measurement of both temporal facilitation and disengagement mechanisms within a single study, the task itself might have blunted observed effects. We matched our dual-task procedure (i.e., identify $\mathrm{T} 1$ and report presence or absence of T2) across mechanisms in order to more directly compare conditions, and also to align with the original task (Raymond et al., 1992). However, matching the mechanisms within the study might have muted the potential effects of disengagement. Whereas the disengagement findings from other studies were observed in single task designs (i.e., T2 task only) that did not require explicit identification of the affective $\mathrm{T} 1$, our explicit $\mathrm{T} 1$ identification task might have diminished differential T1 category effects.

Two other characteristics of our study may have reduced our ability to detect a statistically significant disengagement effect. First, we had adequate power to detect effects that were at least moderately large in magnitude. However, the magnitude of the observed disengagement effects was small-to-medium. Thus, future research should examine the possibility that spider phobia is characterised not only by facilitated detection of threat-relevant stimuli but also by reliable, but smaller magnitude, delayed disengagement from threat-relevant stimuli, although it is important to consider that smaller effects might not translate 
into meaningful real-world mechanisms underlying problematic processing of threat information. The absence of a disengagement effect also might be attributable to a perceptual floor effect. Per cent correct T2 detection in the disengagement block ranged from $65 \%$ to $74 \%$ at the earliest lag across T1 stimulus types, which might have restricted the degree to which spider-phobic participants could display worse performance following spider stimuli relative to negative or neutral stimuli at $\mathrm{T} 1$. Modification of task parameters to enhance performance following neutral or negative stimuli at T1 could provide more room to observe specific effects, and future research might address these parameters.

Three additional issues also merit consideration in future research using the RSVP task to map these temporal mechanisms of attention allocation. First, we presented second targets at only three separate lags after the first target, including the two lags most likely to fall within the $A B$ as well as a lag likely to fall outside the typical $\mathrm{AB}$. These design parameters enhanced our power to investigate snapshots of T2 detection at early lags within the $A B$ window, but precluded the examination of other properties of the $A B$, such as its duration. Second, the photos in each stimulus category were not novel after their initial presentation. Habituation to the spider stimuli might have diminished perceptual effects (e.g., Wright et al., 2000). Our exploratory follow-up analyses stemmed from and supported this possibility, although further investigation is needed. Third, although phobic participants who were eligible for the current study met diagnostic criteria for spider phobia, they did not endorse the highest levels of spider fear, as such potential participants also endorsed extremely high levels of snake fear and thus were excluded from participation. This feature of the sample may have truncated the observed phobia-relevant effects, although it also suggests that the demonstrated facilitation effect is especially robust as it was observed in participants whose symptom severity was not at ceiling.

We note that the general pattern of temporal allocation of attention to threat features in specific phobia differs from the observed pattern of similarly named mechanisms underlying covert and overt spatial allocation of attention (Weierich et al., 2008). As discussed earlier, covert spatial processing of threat is marked by an absence of preferential shifting of covert attention to threat, but marked difficulty with covert disengagement from threat (Weierich et al., 2008). Overtly, early in a perceptual episode, anxious individuals are more likely to move their eyes to threat, but later in the episode they display a tendency to direct their eyes away from threat. In the current study, we addressed the operation of visual attention once the location of threat is attended, and our results suggest that sensitivity to perceptual threat features plays a role in directing attention; once the location is attended, the anxious person is more efficient at identifying a particular percept (e.g., brown spot on wall) as threat (e.g., a spider).

The current results have implications for our understanding of specific phobia and potentially also the other anxiety and stress-related disorders. Whereas much evidence suggests that anxiety disorders are characterised by threat-related “attentional bias" (c.f., Cisler \& Koster, 2010), most of this work does not disambiguate the mechanisms involved in differential attentional processing of threat. The current results suggest the possibility of differential temporal processing of visual threat-relevant information in other conditions for which visual triggers are prominent, such as PTSD. For example, on-going visual processing (i.e., of the road while driving) might be maladaptively interrupted by a glimpse of a threat-relevant percept (i.e., state trooper pointing radar gun) in a veteran with PTSD. On the other hand, disorders marked by more internal processing (e.g., generalised anxiety disorder, GAD) might be characterised more by temporal difficulties that are less dependent on the presence of visual threat information. For example, people with GAD might display prolonged attention to representations of now-absent visual information, which is consistent with recent evidence of a failure to inhibit encoding of non-task threatrelevant information in people with higher trait anxiety (Stout, Shackman, \& Larson, 2013). Pre- and post-treatment measurement of the

COGNITION AND EMOTION, 2015, 29 (6) 1003 
mechanisms of visual processing of threat will help isolate the mechanisms of treatment change. Given exposure-based treatments might act on multiple specific mechanisms of threat processing, a systematic investigation of the problematic mechanisms of visual processing could enhance our ability to tailor exposure-based treatments.

Future research also should more precisely disambiguate the properties of arousal and valence in the perceptual processing of threat. Maladaptive processing in some anxiety disorders might be associated only with those mechanisms that are influenced by arousal (e.g., Vogt, De Houwer, Koster, Van Damme, \& Crombez, 2008), whereas a more general effect of valence might operate in and be adaptive for normative visual processing. Future work also should seek to examine the duration of the $\mathrm{AB}$ related to both the facilitation and disengagement mechanisms by increasing the number of lags measured and by calibrating stimulus parameters to maximise the ability to observe the effect.

In summary, we provide evidence that spider phobia, as a model condition for visual threat processing, is associated with marked differences in the temporal mechanisms of perceptual processing of threat information. People with spider phobia exhibit robustly enhanced facilitation of attention to visual threat features that is independent of baseline processing efficiency and of negative valence, although in this study they did not display difficulty with disengagement from threat stimuli. These data provide a more nuanced view of the nature of threat processing that may contribute to the onset and maintenance of symptoms in specific phobia and anxiety and stress-related disorders more generally.

Manuscript received 13 May 2014

Revised manuscript received 24 August 2014

Manuscript accepted 27 August 2014

First published online 23 September 2014

\section{REFERENCES}

Amir, N., Taylor, C. T., Bomyea, J. A., \& Badour, C. L. (2009). Temporal allocation of attention toward threat in individuals with posttraumatic stress symptoms. Journal of Anxiety Disorders, 23, 10801085. doi:10.1016/j.janxdis.2009.07.010

Anderson, A. K. (2005). Affective influences on the attentional dynamics supporting awareness. Journal of Experimental Psychology, General, 134, 258-281. doi:10.1037/0096-3445.134.2.258

Anderson, A. K., \& Phelps, E. A. (2001). Lesions of the human amygdala impair enhanced perception of emotionally salient events. Nature, 411, 306-309. doi:10.1038/35077083

Arend, I., \& Botella, J. (2002). Emotional stimuli reduce the attentional blink in subclinical anxious subjects. Psicothema, 14, 209-214. Retrieved from http://redalyc2.uaemex.mx/articulo.oa?id=72714204

Arnell, K. M., Killman, K. V., \& Fijavz, D. (2007). Blinded by emotion: Target misses follow attention capture by arousing distracters in RSVP. Emotion, 7, 465-477. doi:10.1037/1528-3542.7.3.465

Barnard, P., Ramponi, C., Battye, G., \& Mackintosh, B. (2005). Anxiety and the deployment of visual attention over time. Visual Cognition, 12, 181-211. doi:10.1080/13506280444000139

Beck, A. T., Steer, R. A., \& Brown, G. K. (1996). Manual for the beck depression inventory (2nd ed.). San Antonio, TX: The Psychological Corporation.

Becker, E. S., \& Rinck, M. (2004). Sensitivity and response bias in fear of spiders. Cognition and Emotion, 18, 961-976. doi:10.1080/02699930341000329

Chaumon, M., Kveraga, K., Barrett, L. F., \& Bar, M. (2013). Visual predictions in the orbitofrontal cortex rely on associative content. Cerebral Cortex. Advance online publication. doi:10.1093/cercor/bht146

Chen, Z. (2012). Object-based attention: A tutorial review. Attention, Perception, \& Psychophysics, 74, 784-802. doi:10.3758/s13414-012-0322-z

Chun, M. M., \& Potter, M. C. (1995). A two-stage model for multiple target detection in rapid serial visual presentation. Journal of Experimental Psychology: Human Perception and Performance, 21, 109127. Retrieved from http://psycnet.apa.org/index. cfm?fa=buy.option ToBuy\&id=1995-20188-001

Cisler, J. M., \& Koster, E. H. (2010). Mechanisms of attentional biases toward threat in anxiety disorders: An integrative review. Clinical Psychology Review, 30, 203-216. doi:10.1016/j.cpr.209.11.003

Cisler, J. M., Ries, B. J., \& Widner, R. L. (2007). Examining information processing biases in spider phobia using the rapid serial visual presentation paradigm. Journal of Anxiety Disorders, 21, 977-990. doi:10.1016/j.janxdis.2006.10.011

\section{4}

COGNITION AND EMOTION, 2015, 29 (6) 
De Jong, P. J., Koster, E. H. W., Van Wees, R., \& Martens, S. (2009). Emotional facial expressions and the attentional blink: Attenuated blink for angry and happy faces irrespective of social anxiety. Cognition and Emotion, 23, 1640-1652. doi:10.1080/02699930 802490227

De Jong, P. J., \& Martens, S. (2007). Detection of emotional expressions in rapidly changing facial displays in high- and low-socially anxious women. Behaviour Research and Therapy, 45, 1285-1294. doi:10.1016/j.brat.2006.10.003

Dux, P. E., \& Marois, R. (2009). The attentional blink: A review of data and theory. Attention, Perception, $E^{2}$ Psychophysics, 71, 1683-1700. doi:10.3758/APP.71. 8.1683

Fox, E., Russo, R., Bowles, R., \& Dutton, K. (2001). Do threatening stimuli draw or hold visual attention in subclinical anxiety? Journal of Experimental Psychology: General, 130, 681-700. Retrieved from http://www.ncbi.nlm.nih.gov/pmc/articles/PMC19 24776/

Fox, E., Russo, R., \& Georgiou, G. A. (2005). Anxiety modulates the degree of attentive resources required to process emotional faces. Cognitive, Affective, and Behavioral Neuroscience, 5, 396-404. Retrieved from http://www.ncbi.nlm.nih.gov/pubmed/16541810

Fredrikson, M., Annas, P., Fischer, H., \& Wik, G. (1996). Gender and age differences in the prevalence of specific fears and phobias. Behaviour Research and Therapy, 34, 33-39. Retrieved from http://www-ncbinlm-nih-gov.proxy.wexler.hunter.cuny.edu/pubmed/ 8561762

Keil, A., \& Ihssen, N. (2004). Identification facilitation for emotionally arousing verbs during the attentional blink. Emotion, 4(1), 23-35. doi:10.1037/1528-3542.4.1.23

Klein, C., Arend, I. C., Beauducel, A., \& Shapiro, K. L. (2011). Individuals differ in the attentional blink: Mental speed and intra-subject stability matter. Intelligence, 39, 27-35. doi:10.1016/j.intell.2010.11.004

Klorman, R., Weerts, T. C., Hastings, J. E., Melamed, B. G., \& Lang, P. J. (1974). Psychometric properties of some specific fear questionnaires. Behavior Therapy, 5, 401-409. Retrieved from http://www.sciencedirect.com/science/article/pii/S0005789474800080

MacLean, M. H., \& Arnell, K. M. (2012). A conceptual and methodological framework for measuring and modulating the attentional blink. Attention, Perception, and Psychophysics, 74, 1080-1097. doi:10.3758/s13414012-0338-4

Maratos, F. A., Mogg, K., \& Bradley, B. P. (2008). Identification of angry faces in the attentional blink.
Cognition and Emotion, 22, 1340-1352. doi:10.1080/ 02699930701774218

Marks, I. M., \& Mathews, A. M. (1979). Brief standard self-rating for phobic patients. Behaviour Research and Therapy, 17, 263-267. Retrieved from http:// www.sciencedirect.com.proxy.wexler.hunter.cuny.edu/ science/article/pii/000579677990041X

Martin, E. W., \& Shapiro, K. L. (2008). Does failure to mask T1 cause lag-1 sparing in the attentional blink? Perception and Psychophysics, 70, 562-570. doi:10.3758/ PP.70.3.562

Mathews, A., \& Mackintosh, B. (1998). A cognitive model of selective processing in anxiety. Cognitive Therapy and Research, 22, 539-560. Retrieved from http://link.springer.com/article/10.1023\%2FA\%3A1 018738019346\#page-1

Mathews, A., \& MacLeod, C. (1994). Cognitive approaches to emotion and emotional disorders. Annual Review of Psychology, 45, 25-50. doi:10.1146/ annurev.ps.45.020194.000325

Mathewson, K. J., Arnell, K. M., \& Mansfield, C. A. (2008). Capturing and holding attention: The impact of emotional words in rapid serial visual presentation. Memory E Cognition, 36, 182-200. doi:10.3758/MC.36.1.182

Mogg, K., \& Bradley, B. (2003). Selective processing of nonverbal information in anxiety: Attentional biases for threat. In P. Philippot, R. Feldman, \& E. Coats (Eds.), Nonverbal behavior in clinical settings (pp. 127-143). New York, NY: Oxford University Press.

Most, S. B., Chun, M. M., Widders, D. M., \& Zald, D. H. (2005). Attentional rubbernecking: Cognitive control and personality in emotion-induced blindness. Psychonomic Bulletin and Review, 12, 654-661. Retrieved from http://link.springer.com/article/10.37 58\%2FBF03196754\#page-1

Most, S. B., Smith, S. D., Cooter, A. B., Levy, B. N., \& Zald, D. H. (2007). The naked truth: Positive, arousing distracters impair rapid target perception. Cognition and Emotion, 21, 964-981. doi:10.1080/ 02699930600959340

Muris, P., \& Merckelbach, H. (1996). A comparison of two spider fear questionnaires. Journal of Behavior Therapy and Experimental Psychiatry, 27, 241-244. Retrieved from http://www.sciencedirect.com.proxy. wexler.hunter.cuny.edu/science/article/pii/S00057916 96000225\#

Ogawa, T., \& Suzuki, N. (2004). On the saliency of negative stimuli: Evidence from attentional blink. Japanese Psychological Research, 46(1), 20-30. doi:10.1111/j.1468-5884.2004.00233.x

COGNITION AND EMOTION, 2015, 29 (6) 
Olatunji, B. O., Armstrong, T., McHugo, M., \& Zald, D. H. (2013). Heightened attentional capture by threat in veterans with PTSD. Journal of Abnormal Psychology, 122, 397-405. doi:10.1037/ a0030440

Olatunji, B. O., Ciesielski, B. G., \& Zald, D. H. (2011). A selective impairment in attentional disengagement from erotica in obsessive-compulsive disorder. Progress in Neuropsychopharmacology and Biological Psychiatry, 35, 1977-1982. doi:10.1016/j. pnpbp.2011.07.005

Panichello, M. F., Cheung, O. S., \& Bar, M. (2013). Predictive feedback and conscious visual experience. Frontiers in Psychology, 3, 1-8. doi:10.3389/fpsyg. 2012.00620

Raymond, J. E., Shapiro, K. L., \& Arnell, K. M. (1992). Temporary suppression of visual processing in an RSVP task: An attentional blink? Experimental Psychology and Human Perception, 18, 849-860. doi:10.1037/0096-1523.18.3.849

Reinecke, A., Rinck, M., \& Becker, E. S. (2008). How preferential is the preferential encoding of threatening stimuli? Working memory biases in specific anxiety and the attentional blink. Journal of Anxiety Disorders, 22, 655-670. doi:10.1016/j.janxdis.2007. 06.004

Rokke, P. D., Arnell, K. M., Koch, M. D., \& Andrews, J. T. (2002). Dual-task attention deficits in dysphoric mood. Journal of Abnormal Psychology, 111, 370-379. doi:10.1037/0021-843X.111.2.370

Schonenberg, M., \& Abdelrahman, T. (2013). In the face of danger: Exploring the attentional blink to emotional facial expressions in PTSD. Psychiatry Research, 209, 180-185. doi:10.1016/j. psychres.2012.11.011

Smith, S. D., Most, S. B., Newsome, L., \& Zald, D. H. (2006). An emotion-induced attentional blink elicited by aversively conditioned stimuli. Emotion, 6, 523-527. doi:10.1037/1528-3542.6.3.523

Spielberger, C. D., Gorsuch, R. L., \& Lushene, R. E. (1970). STAI manual for the state-trait anxiety inventory. Palo Alto, CA: Consulting Psychologists Press.

Stout, D. M., Shackman, A. J., \& Larson, C. L. (2013). Failure to filter: Anxious individuals show inefficient gating of threat from working memory. Frontiers in Human Neuroscience, 7, 58. doi:10.3389/fnhum.2013. 00058

Szymanski, J., \& O’Donohue, W. (1995). Fear of spiders questionnaire. Journal of Behavior Therapy and Experimental Psychiatry, 26(1), 31-34. Retrieved from http:// www.sciencedirect.com.proxy.wexler.hunter.cuny.edu/ science/article/pii/000579169400072T

Tibboel, H., Van Bockstaele, B., \& De Houwer, J. (2011). Is the emotional modulation of the attentional blink driven by response bias? Cognition and Emotion, 25, 1176-1183. doi:10.1080/02699931. 2010.524192

Trippe, R. H., Hewig, J., Heydel, C., Hecht, H., \& Miltner, W. H. R. (2007). Attentional blink to emotional and threatening pictures in spider phobics: Electrophysiology and behavior. Brain Research, 1148, 149-160. doi:10.1016/j.brainres.2007.02.035

Vogt, J., De Houwer, J., Koster, E. H. W., Van Damme, S., \& Crombez, G. (2008). Allocation of spatial attention to emotional stimuli depends upon arousal and not valence. Emotion, 8, 880-885. doi:10.1037/a0013981

Weierich, M. R., Treat, T. A., \& Hollingworth, A. (2008). Theories and measurement of visual attentional processing in anxiety. Cognition and Emotion, 22, 985-1018. doi:10.1080/02699930701597601

Wright, C. I., Fischer, H., Whalen, P. J., McInerney, S. C., Shin, L. M., \& Rauch, S. L. (2000). Differential prefrontal cortex and amygdala habituation to repeatedly presented emotional stimuli. NeuroReport, 12, 379-383. Retrieved from http://www-ncbi-nlm-nihgov.proxy.wexler.hunter.cuny.edu/pubmed/11209954

Zhang, J., \& Mueller, S. T. (2005). A note on ROC analysis and non-parametric estimate of sensitivity. Psychometrika, 70, 203-212. doi:10.1007/s11336003-1119-8 